BMC

Research Notes
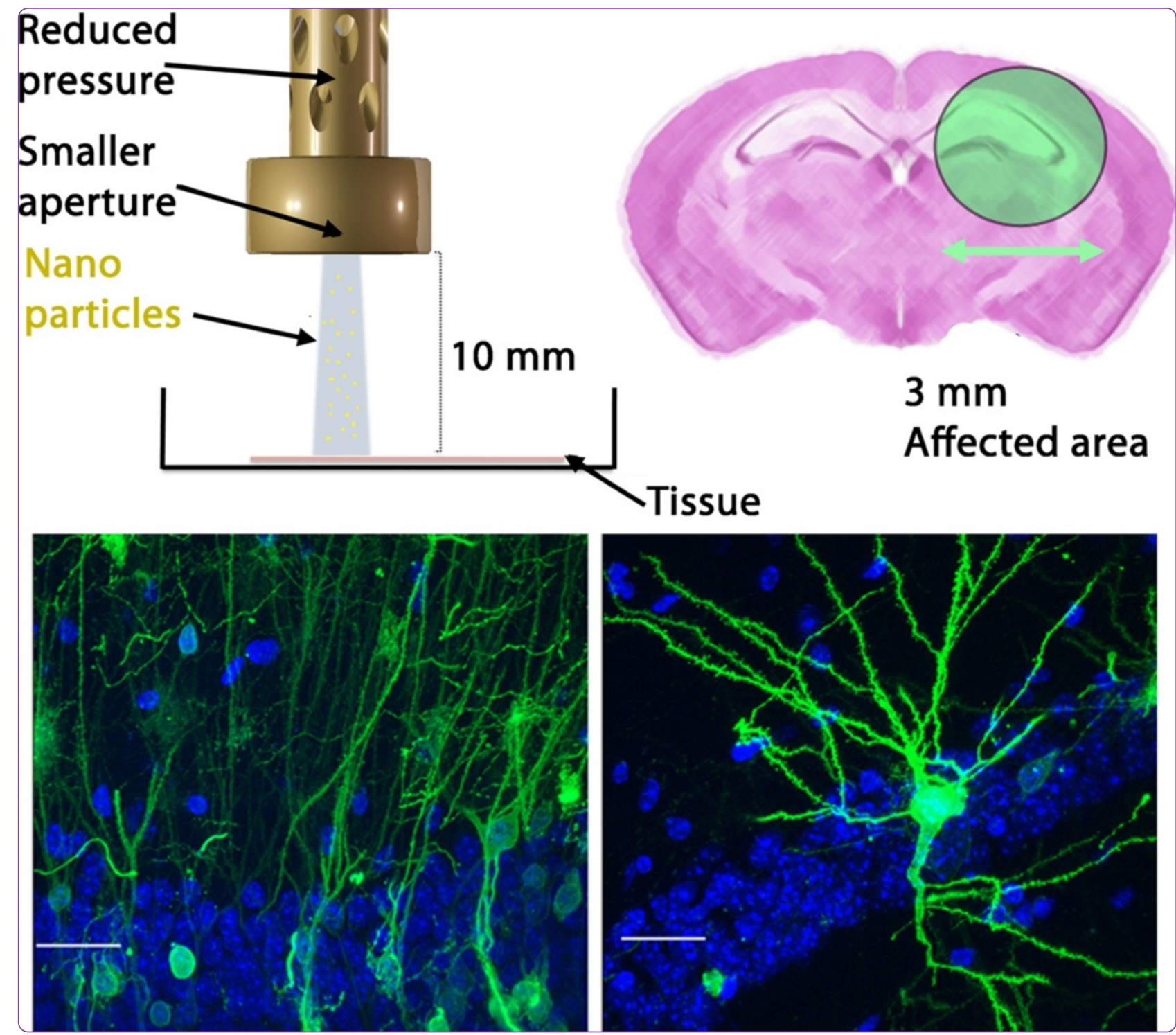

\title{
Optimized heterologous transfection of viable adult organotypic brain slices using an enhanced gene gun
}

Arsenault and O'Brien 


\title{
Optimized heterologous transfection of viable adult organotypic brain slices using an enhanced gene gun
}

Jason Arsenault and John A O’Brien ${ }^{*}$

\begin{abstract}
Background: Organotypic brain slices (OTBS) are an excellent experimental compromise between the facility of working with cell cultures and the biological relevance of using animal models where anatomical, morphological, and cellular function of specific brain regions can be maintained. The biological characteristics of OTBS can subsequently be examined under well-defined conditions. They do, however, have a number of limitations; most brain slices are derived from neonatal animals, as it is difficult to properly prepare and maintain adult OTBS. There are ample problems with tissue integrity as OTBS are delicate and frequently become damaged during the preparative stages. Notwithstanding these obstacles, the introduced exogenous proteins into both neuronal cells, and cells imbedded within tissues, have been consistently difficult to achieve.
\end{abstract}

Results: Following the ex vivo extraction of adult mouse brains, mounted inside a medium-agarose matrix, we have exploited a precise slicing procedure using a custom built vibroslicer. To transfect these slices we used an improved biolistic transfection method using a custom made low-pressure barrel and novel DNA-coated nanoparticles $(40 \mathrm{~nm})$, which are drastically smaller than traditional microparticles. These nanoparticles also minimize tissue damage as seen by a significant reduction in lactate dehydrogenase activity as well as propidium iodide (PI) and dUTP labelling compared to larger traditional gold particles used on these OTBS. Furthermore, following EYFP exogene delivery by gene gun, the $40 \mathrm{~nm}$ treated OTBS displayed a significantly larger number of viable NeuN and EYFP positive cells. These OTBS expressed the exogenous proteins for many weeks.

Conclusions: Our described methodology of producing OTBS, which results in better reproducibility with less tissue damage, permits the exploitation of mature fully formed adult brains for advanced neurobiological studies. The novel $40 \mathrm{~nm}$ particles are ideal for the viable biolistic transfection of OTBS by reducing tissue stress while maintaining long term exogene expression.

Keywords: Organotypic brain slices, Vibroslicer, Gene gun, Biolistic transfection, Nanoparticles, Tissue slicer

\section{Background}

Organotypic brain slices (OTBS) are viable segments of brain tissue that can be cultured in vitro. Improvements in brain slice methodology are required to permit this strategy to be utilized more frequently for a wide range of biological applications. Of particular advantage is the fact that OTBS maintain many characteristics of in vivo biology including functional synaptic circuitry and also preserve local brain architecture $[1,2]$. Brain slices are increasingly being used

\footnotetext{
* Correspondence: jaob@mrc-Imb.cam.ac.uk

Neurobiology Division, MRC Laboratory of Molecular Biology, Francis Crick Avenue, Cambridge CB2 OQH, UK
}

for both basic and applied research, and have been proven successful for a number of pharmacological and genetic manipulations that investigate particular neurobiological functions [3-7]. Many biological questions can't be sufficiently addressed using cell cultures while whole animal in vivo studies aren't permissive to numerous biotechnological assays [6,7]. OTBS maintain advantages of both.

OTBS have successfully been established on a variety of brain regions including hippocampus, hypothalamus, striatum, cortex, spinal cord, and cerebellum [8-14]. For further reading on the full range of OTBS regions studied please read the review by Lossi et al., 2009 [2]. In addition, a range of tissue slice co-cultures have also been reported, 
which allow the assessment of neuronal intercommunication across brain regions. The utilities of these co-cultures have been well established with the examples of hippocampal, cortico-spinal, and cortico-striatal preparations [15-19] as well as testing OTBS under the effects of other cells such as carcinomas [20]. Several methods have been developed to maintain thin OTBS under long-term culture $[21,22]$ based on the use of "roller tubes" or Maximovtype chambers, although preparing reproducible cultures using the conventional roller tube technique is especially demanding, and such cultures often display considerable experimental variability. Following these, a membrane interface culture method was described [23], which allows straightforward solution changes and easy access to the slices. This can be very advantageous, and many studies now use a modification of this membrane interface method. For a more indebt historical review of alternative neuronal culture methods please read Millet and Gillette, 2012 [1]. However, the preparation of experimentally reliable slices is still a major obstacle, and successfully viable adult OTBS are exceptionally rare [2,24-26].

Another major hurdle in the consistent use of OTBS is their intransigence with DNA transfection. Terminally differentiated cells such as neurons are difficult to transfect themselves, while cells within tissue aren't easily exposed to the administered reagents. A potential solution to this is biolistic transfection, where genetic material, coated onto micro or nanoparticles, is fired through tissue, propelled by the pressurized gas of a "gene gun" [27]. This, however, can cause considerable tissue damage, exacerbating what might already have arisen from the handling of delicate OTBS. For these reasons further advancements are thus required to expand the utility of OTBS.

Here we describe an improved method of preparing adult OTBS with increased ease of preparation. These adult OTBS were then used to explore the efficiency of a modified biolistic transfection procedure using novel and less damaging, nanometer-sized DNA bound gold particles fired by a lower pressure and more precise barrel. These fired nano-particles, while concomitantly heightening the precision of the transfected area $(\sim 3 \mathrm{~mm}$ diameter) compared to traditional gene gun, preserve cellular viability and also elevate transfection yields within these difficult to transfect cells.

\section{Results}

Agarose-embedded brain slices

To preserve the integrity of the tissue, the brains were embedded in an agarose block (Figure 1A). As the brain is placed in the ice-cold agarose solution as soon as it is removed, and the agarose block is constructed with cellfriendly Dulbecco's modified eagle medium (DMEM) containing penicillin and streptomycin; degradation and potential contamination of the tissue were thus minimized.

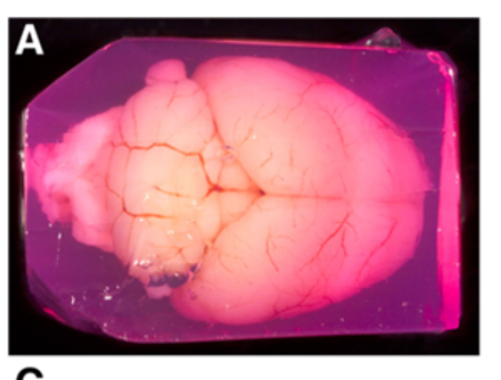

C

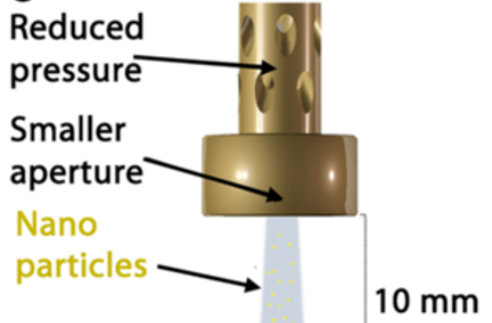

B

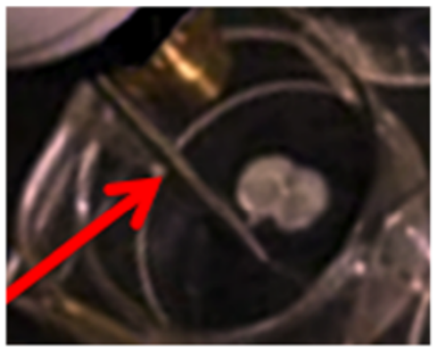

D

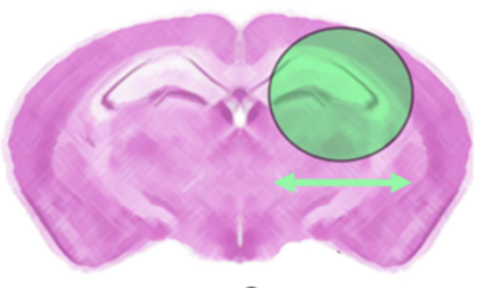

$3 \mathrm{~mm}$

Affected area

Figure 1 Adult organotypic brain slice preparation and biolistic transfection. A) The adult mouse brain were embedded in DMEM-agarose with penicillin/streptomycin. This DMEM-agarose matrix, readily suitable for handling, contains phenol red, hence the red coloration. This colour gradient also helps to see the OTBS cut by the vibroslicer. Scale bar: $10 \mathrm{~mm}$. B) The OTBS seen in an empty well ready for transfection by biolistics. A red arrow indicates a rod support used to maintain distance and angle. C) Scaled model showing the height of the modified barrel over the OTBS. D) Distribution pattern of the scattered micro- and nanoparticles. The affected and chosen region of interest subsequently expresses the exogene [29]. This biolistic transfection method is ideally suited for adult OTBS. 
More importantly, the brain imbedded in the mediumagarose matrix becomes easier to slice, and these subsequent OTBS can easily be manipulated and this maintains the regional brain anatomy undamaged. Viability of the OTBS is also improved by the slicing procedure due to its minimal handling. The vibroslicer (Additional file 1: video), which we had constructed from details described by [28], is particularly appropriate for these adult brains as slicing is performed with large-amplitude and high frequency horizontal oscillations; these allow for proficient cutting of sticky myelinated fibers, of which there are an abundance in adult brain. Since vertical vibrations of the blade are minimal, typically $0.5-1.5 \mu \mathrm{m}$, this avoids waves of damaging compressions to the superficial tissue layers. Microscopic examination of our slices indicated that cellular and subcellular elements were largely intact $20 \mu \mathrm{m}$ below the surface (as determined by Z scanning; data not shown). Our procedure allowed the slices to remain qualitatively viable in culture for up to 6 weeks as abundant PI negative and DAPI positive cells could still be observed within the OTBS that also maintained structural integrity (data not shown).

\section{Nanoparticle mediated biolistic transfection and cell survival}

Efficient transfection of OTBS is difficult using traditional techniques but we have previously shown that biolistic transfection using a modified gene gun (Figure 1B-D) can result in efficient and precisely localized gene expression [27]. However, we and others have showed that biolistic transfection can cause tissue damage, which is a significant problem for these delicate adult OTBS, see [27] and refs therein.

As was suggested by a previous study, the use of smaller gold particles might reduce inherent tissue stresses [29]. Here we further tested the use of nanoparticles (1/25th the scale of standard particles) to deliver the genetic material, and the OTBS displayed limited cell damage compared to $1 \mu \mathrm{m}$ particles. Figure $2 \mathrm{~A}$ shows total survival rates of untreated, $40 \mathrm{~nm}$, and $1 \mu \mathrm{m}$ particles treated OTBS as revealed by LDH assay. There was no difference observed between untreated and $40 \mathrm{~nm}$ biolistics transfected cells whereas a significant reduction in viability in the $1 \mu \mathrm{m}$ particle treated OTBS was seen 5 days after treatment $\left({ }^{*} p<0.05\right)$. PI labelling displayed a similar trends (Figure $2 \mathrm{~B}$ ) where the $40 \mathrm{~nm}$ particle treated OTBS displayed no discernable increase in the number of cells stained with this necrosis marker while the $1 \mu \mathrm{m}$ particles significantly elevated the number of necrotic cells. dUTP labelling, a typical apoptosis marker, showed a slight increase in the $40 \mathrm{~nm}$ particle treated OTBS while a substantial increase in the $1 \mu \mathrm{m}$ particle treated OTBS was observed (Figure 2C). Overall these analyses consistently indicate that the $40 \mathrm{~nm}$ particles are much less damaging to the OTBS than the $1 \mu \mathrm{m}$ particles.

\section{Lineage quantification within the OTBS and exogene expression}

In order to appropriately exploit these OTBS we measured population differences between glial and neuronal cells 5 days following transfection in the vicinity of the CA1 region. Figure 2D shows the counts of cells immunolabelled with NeuN/Fox-3, a widely used neuronal marker, in comparison to glial fibrillary acidic protein (GFAP), characteristically associated to the glial lineage. The number of $\mathrm{NeuN}$ positive cells counted within comparable brain regions of the corresponding coronal sections showed a significant reduction $\left({ }^{*} \mathrm{p}<0.05\right)$ in the number of neurons present following the $1 \mu \mathrm{m}$ particles treatment compared to the $40 \mathrm{~nm}$ particles treated OTBS. However, the number of glial cells remained similar, indicating that the fragile neuronal cells were more sensitive to the $1 \mu \mathrm{m}$ particle penetration. Figure $3 \mathrm{~A}$ shows representative images of OTBS labelled with anti-NeuN, anti-GFAP, and DAPI at 5 and 14 days following treatment. As can be seen in Figure 3A, we have also observed GFAP positive cells proliferating along the outer edges that occurred irrespective of gold particle size used [30]. Overall, cells transfected using $40 \mathrm{~nm}$ particles maintain normal morphology and the expression of the immunological marker NeuN longer than the cells treated with $1 \mu \mathrm{m}$ particles.

Transfection rates as determined by the number of EYFP positive cells over the total amount of DAPI positive cells were also more elevated in the $40 \mathrm{~nm}$ particle treated OTBS compared to $1 \mu \mathrm{m}$ particles and this expression can still be abundantly observed in terminally differentiated neuronal cells (Figure 2E). This expression pattern can be seen at 5,14 , and 21 days for the $40 \mathrm{~nm}$ and $1 \mu \mathrm{m}$ particle treated OTBS in Figure 3B.

\section{Highly detailed images of fully formed adult neurons}

Biolistic transfection using $40 \mathrm{~nm}$ EYFP-DNA gold particles, which resulted in significantly reduced tissue damage compared to microparticles, showed brilliant expression patterns in whole cells.

As can be seen in Figure 4, bright neurites of individually cells can be mapped after transfection. Figure 4A shows two parallel pyramidal cells in the hippocampus. In Figure 4B, the polarized axonal distribution shown in the CA1 hippocampal region, which is anatomically representative of this brain structure, can be clearly observed. Figure $4 \mathrm{C}$ shows another pyramidal cell, where fully formed mature dendrites radiate axially towards their respective synaptic interface.

\section{Discussion}

Here we describe an improved method of preparing OTBS for biolistic transfection. The efficient medium-agarose embedded strategy, results in excellent viability due to ease of handling, and thus can be used for both immature 

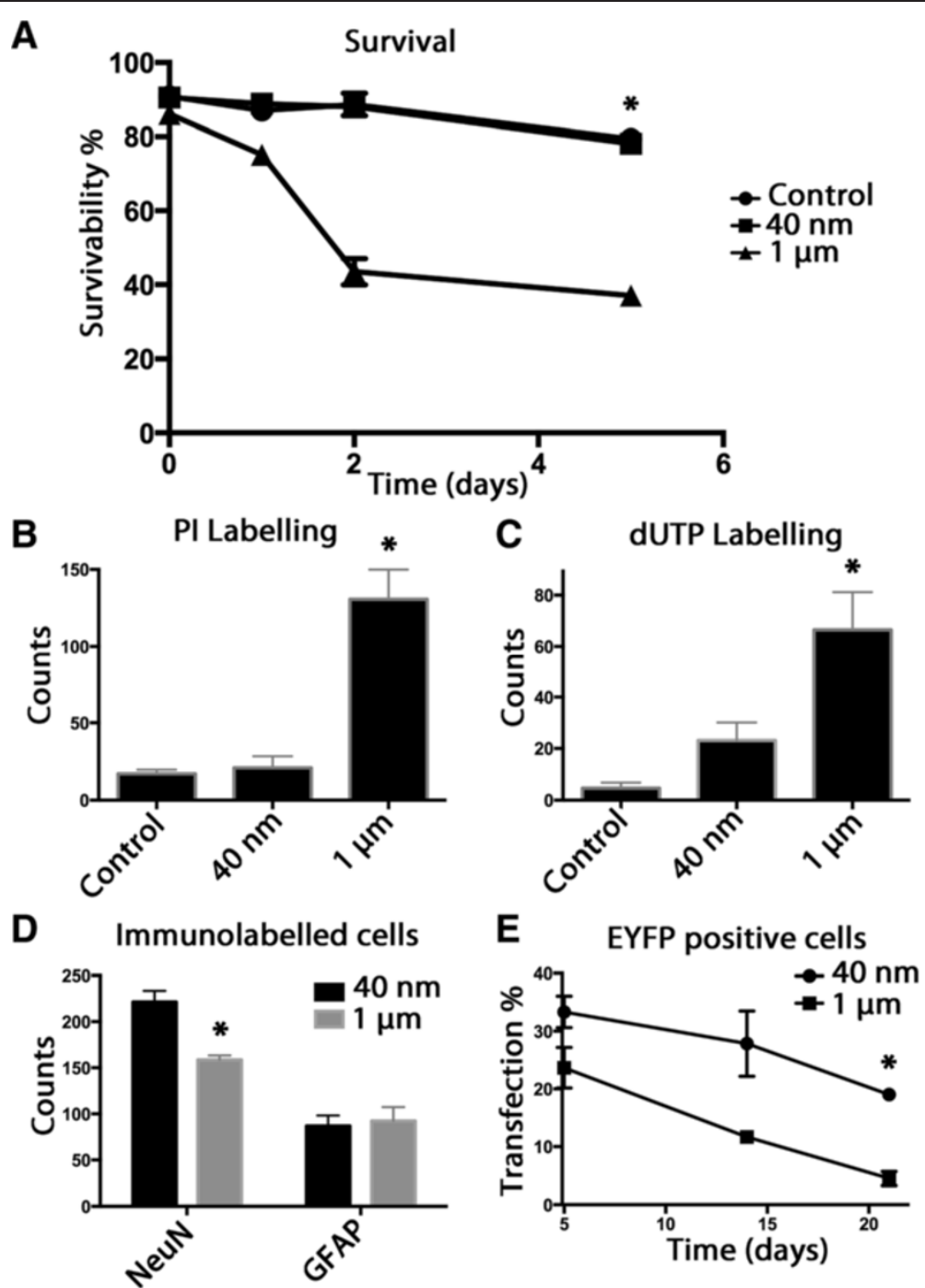

Figure 2 Organotypic brain slice viability, cellular populations, and exogene expression profile. A) Lactate dehydrogenase assay used to monitor cell viability. A significant reduction of cell viability in the $1 \mu \mathrm{m}$ particle treated OTBS was measured using the LDH assay can be observed 5 days following treatment compared to control and $40 \mathrm{~nm}$ treated OTBS $\left(n=5,{ }^{*} p<0.05\right)$. B) PI labelling at 5 days post transfection shows a significant elevation of the number of necrotic cells in the $1 \mu \mathrm{m}$ treated OTBS compared to $40 \mathrm{~nm}$ treated and untreated OTBS ( $n=6$, $\left.{ }^{*} \mathrm{p}<0.05\right)$ as seen in representative brain regions. C) dUTP TUNEL assay at 5 days post transfection shows a slight elevation of nick end labelling in the $40 \mathrm{~nm}$ treated OTBS while a significant elevation in the number of dUTP positive cells compared to the untreated control can be observed in the $1 \mu \mathrm{m}$ treated OTBS $\left(n=6,{ }^{*} p<0.05\right)$. D) anti-NeuN and anti-GFAP immunolabelling at 5 days post transfection was used to quantify neuronal and glial populations respectively. A significant reduction in the number of NeuN positive cells was observed in comparable visual fields of the CA1 region of the hippocampus of corresponding coronal sections for the $1 \mu \mathrm{m}$ compared to the $40 \mathrm{~nm}$ particle treated OTBS $\left(n=6,{ }^{*} p<\right.$ 0.05). E) EYFP expression patterns seen in the $40 \mathrm{~nm}$ and $1 \mu \mathrm{m}$ treated OTBS from 5 to 21 days after transfection. A significantly higher percentage of cells remained EYFP positive three weeks after transfection in the $40 \mathrm{~nm}$ treated OTBS compared to the $1 \mu \mathrm{m}$ treated OTBS ( $n=6,{ }^{*} p<$ 0.05) co-labelled with DAPI.

and myelinated adult brains. We also demonstrate that using biolistic transfection with smaller, nanometer-sized particles combined with the modified gun barrel, also minimizes the damage to OTBS, which ultimately heightens exogenous protein expression inside cells that have consistently been shown refractory to DNA transfection.

Typically OTBS are prepared from the brains of animals at postnatal days 3-9. These brains show a high degree of plasticity and resistance to mechanical trauma during the preparation, which has proved important to obtain viable and healthy cultures. Although at this age basic synaptic connections have been established in a number of brain regions (e.g. in the CA1 area of the hippocampus), the mature synapses have not yet fully developed $[31,32]$. Thus using pup brain is less than ideal to study many aspects of synaptic physiology where adult brain would be necessary, 


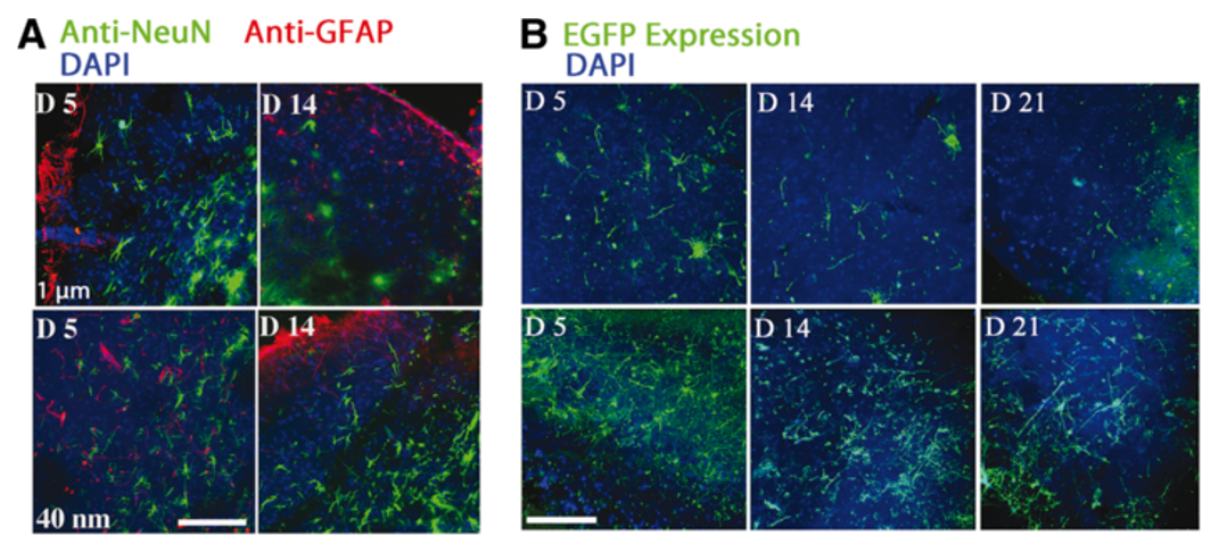

Figure 3 Microscopy images of subpopulation labelling and exogene expression profiles. A) Confocal images of the $40 \mathrm{~nm}$ and $1 \mu \mathrm{m}$ treated OTBS immunolabelled with anti-NeuN, anti-GFAP, and DAPI seen at 5 and 14 days post transfection. Healthier cell morphologies can be observed in the $40 \mathrm{~nm}$ compared to the $1 \mu \mathrm{m}$ treated OTBS. Scale bar: $100 \mu \mathrm{m}$. B) Confocal images of EYFP expression patterns seen in $40 \mathrm{~nm}$ and $1 \mu \mathrm{m}$ transfected OTBS. A higher number of EYFP positive cells, maintaining neuronal morphology, can be observed in the $40 \mathrm{~nm}$ compared to $1 \mu \mathrm{m}$ treated OTBS and this expression pattern remains longer (21 days). Scale bar: $100 \mu \mathrm{m}$.

e.g. "connectomics", age-related neurodegeneration, adult neurogenesis [33-36].

Even in neonatal OTBS that maintain sufficient viability, there arises a gradual loss of morphological characteristics that therefore doesn't sufficiently represent the brain regions under long-term study [37]. This loss of characteristics might be due to the more pronounced cellular dedifferentiation occurring in neonatal cultures compared to older animal OTBS - a hypothesis supported by several studies [38,39]. Reproducible adult OTBS are difficult to obtain, but the DMEM-agarose embedding procedures in combination with a stable vibroslicer promote the use of adult OTBS. The vibroslicer greatly facilitates the cutting of the brain into slices which are then effortlessly separated during the slicing procedure, as opposed to a tissue chopper where difficulties in separating very thin $(150 \mu \mathrm{m})$ and sticky adult OTBS often occurs (slices can adhere both to the blade and to themselves while using a tissue chopper). This reduced thickness of the OTBS that facilitates the absorption of nutrient from the medium was also supported by other studies [33]. Variations of culture medium supplementation for adult OTBS have also been shown encouraging [24]. The vibroslicer essentially minimizes any additional stresses to the tissue. Although our instrument was custom designed, there are now great quality commercially available vibroslicers (e.g. World Precision Instruments Model No. NVSL and Leica VT 1000S). Since it was impossible to prepare adult $150 \mu \mathrm{m}$ OTBS using a tissue chopper, the comparison between slicing methods could not be addressed in this study. Nevertheless, ease of manipulation inherently reduces stresses to, and promotes the preservation of, these fragile tissues.

The efficient transfection of genetic material into OTBS has tremendous potential, yet, lacking in sufficient
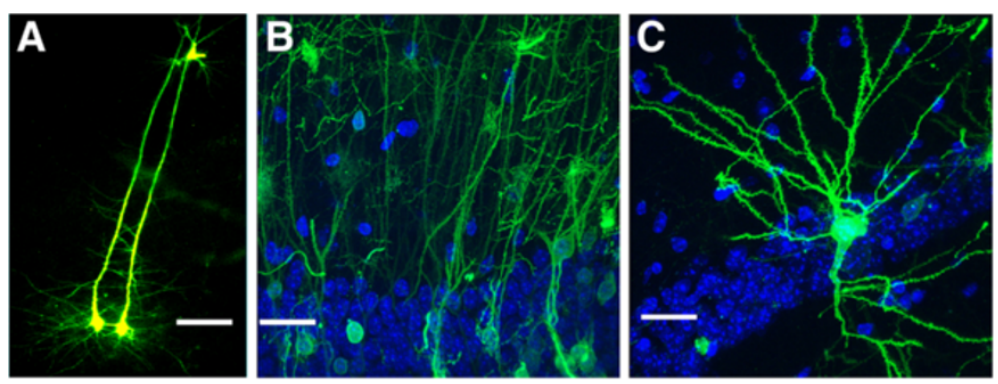

Figure 4 Observing morphological characteristics in mature neurons of adult OTBS. A) Confocal image of neighbouring pyramidal cells, which were treated with the $40 \mathrm{~nm}$ particles, shows long axons oriented towards the cortex as seen by EYFP expression at 5 days post transfection. Scale bar: $25 \mu \mathrm{m}$. B) Another confocal image showing a population of pyramidal cells having their soma present in the CA1 region of the brain 5 days following EYFP transfection with the $40 \mathrm{~nm}$ particles. The radiating arborescence of neurites formed within the adult brain can clearly be seen. Cells were also stained with DAPI. Scale bar: $10 \mu \mathrm{m}$. C) Confocal image of another pyramidal cell 5 days following transfection with $40 \mathrm{~nm}$ particles showing EYFP expression. The soma also rests within the densely populated CA1 region of the hippocampus while the neurites branch out towards their respective synaptic interfaces. Cells were also stained with DAPI. Scale bar: $10 \mu \mathrm{m}$. This transfection procedure adequately preserves the morphological characteristics and architecture of mature neurons within these ex vivo adult OTBS. 
advancement, this approach has had limited success. There are many methods of inserting DNA into cultured cells, including lipofection, electroporation, viral transduction, and microinjection [40-42]. However, none of these are ideal for OTBS, largely due to the poor DNA uptake into the nucleus of terminally differentiated cells such as neurones, and this is further compounded by the lack of access to cells deep within the tissue. Biolistic transfection overcome both hindrances, but can cause significant tissue damage, which can be exasperatingly problematic with delicate adult OTBS. Our recent work [29] suggested that nanometer sized DNA coated particles are as efficient as the widely used micrometer sized particles do deliver genetic material, yet could minimize incidental damages, suggested that this approach would be ideal for OTBS and other preparations. Our current data supports this hypothesis and shows improved viability of the cells in the OTBS following genetic delivery. These adult OTBS also showed exquisite details of neural architecture that were preserved and these can subsequently be meticulously investigated. The observed morphology of these neuronal cells can thus be analysed by various methods such as measuring neurites or using a combination of fluophores, stochastically delivered, to visualise intercellular synaptic networks $[7,43,44]$. Overall these results show that this improved protocol to prepare adult OTBS are ideal for biolistic transfection, that the $40 \mathrm{~nm}$ DNA coated gold particles minimizing cell and tissue damage, and that the exogene can remains expressed for weeks.

\section{Conclusions}

This improved method of producing finely sliced adult OTBS for biolistic transfection and exploiting novel nanometer-sized particles allows for the efficient expression of exogenous proteins while minimizing tissue damage, which is essential to properly visualize the morphological, anatomical, and cellular functions within fully formed adult brains structures. These improvements in OTBS preparation in conjunction with the modified gene gun becomes a useful platform for in vitro transfection of ex vivo organotypic slices and enhances their possible applications for subsequent genetic and biochemical manipulations. These results also encourage further translational expansion of the biolistic delivery methods for medical and bio-technological applications.

\section{Methods}

\section{Brain/agarose preparation}

Six week old C57 Black 6 mice were sacrificed according to ethical committee approval and their brains dissected as previously described [45]. A solution of $2 \%$ LMP agarose was prepared in Dulbecco's modified eagles medium (DMEM) supplemented with $100 \mathrm{U} / \mathrm{ml}$ penicillin and $100 \mu \mathrm{g} / \mathrm{ml}$ streptomycin $(\mathrm{P} / \mathrm{S})$, and cooled $\left(4-10^{\circ} \mathrm{C}\right)$. The freshly removed brain was then placed in a plastic mould and covered with the agarose solution; the mould was then rapidly cooled to $4^{\circ} \mathrm{C}$ on ice. When the agarose set $(5-10 \mathrm{~min})$, the medium-agarose embedded brain (Figure 1A) was removed from the mould, trimmed if necessary, and then super glued (Loctite) to a copper base platform. This was then placed into the vibroslicer chamber that contained ice-cold phosphate buffered saline (PBS: $137 \mathrm{mM} \mathrm{NaCl}, 2.7 \mathrm{mM} \mathrm{KCl}$, $8.1 \mathrm{mM} \mathrm{Na}_{2} \mathrm{HPO}_{4}, 1.76 \mathrm{mM}, \mathrm{KH}_{2} \mathrm{PO}_{4}, \mathrm{pH}$ 7.4).

\section{Slice preparation}

Slices were prepared using a vibroslicer as previously described [28]. The vibroslicer was constructed by the engineers at the Laboratory of Molecular Biology's mechanical workshop using these specifications [28]. Briefly, the oscillation frequency was $90 \mathrm{~Hz}$, the amplitude $1.5-2.0 \mathrm{~mm}$, and the cutting blade positioned at a $15^{\circ}$ angle to the horizontal plane; the mounting block bearing the mediumagarose embedded brain was set to move at $1.7 \mathrm{~mm} / \mathrm{min}$ towards the blade. Sections $(150 \pm 1.5 \mu \mathrm{m})$ were collected into a falcon containing ice-cold culture media (DMEM, $1 \% \mathrm{P} / \mathrm{S}$, and $10 \%$ Fetal Calf Serum (FCS)). See Additional file 1: video. The slices were then placed into $0.4 \mu \mathrm{m}$, $30 \mathrm{~mm}$ diameter cell culture inserts (Milli Cell) in a 6 multi-well tray with culture media on the outside of the insert, and incubated in a humidified incubator at $37^{\circ} \mathrm{C}$ with $5 \% \mathrm{CO}_{2}$. The coronal brain slices were carefully positioned in the centre of the insert and the insert positioned to orient the brain's dorsal region the same way in each well.

\section{Preparation of micro/nano particles}

Particles were prepared as described [27] using $1 \mu \mathrm{m}$ or $40 \mathrm{~nm}$ diameter gold particles. Briefly, $50 \mu \mathrm{l}$ of $0.05 \mathrm{M}$ spermidine and $10 \mu \mathrm{l}$ DNA at $1 \mathrm{mg} / \mathrm{ml}$ (pEYFP-N1; Clontech, USA) were added to $10 \mathrm{mg}$ of particles. These were mixed while adding $50 \mu \mathrm{l}$ of $1 \mathrm{M} \mathrm{CaCl}_{2}$ in $10-15 \mu \mathrm{l}$ drops. After 5 min with occasional mixing, the supernatant was removed by centrifugation $(1,000 \times \mathrm{g}$ for $30 \mathrm{~s})$ and the gold pellet resuspended in $3.5 \mathrm{ml} 0.075 \mathrm{M}$ polyvinylpyrrollidone (PVP; Sigma). This suspension was then inserted into Tefzel tubing ( $0.1 \mathrm{~mm}$ internal diameter; Bio-Rad) using a syringe. The tubing was placed in the tubing preparation station (Bio-Rad), the gold particles were allowed to settle, and the supernatant removed with a syringe. Then the tubing was rotated to ensure an even spread of gold particles, which were subsequently dried under nitrogen flow. To create bullets, the tubing was cut using a tubing cutter (Bio-Rad) into $1 \mathrm{~cm}$ lengths, which were either immediately inserted into the gene gun cartridge, or kept desiccated at $4^{\circ} \mathrm{C}$ until required.

\section{Biolistic transfection}

Brain slices were transfected with biolistics as previously described [29] using a gas pressure of $50 \mathrm{psi}$ at a distance 
of $10 \mathrm{~mm}$ (Figure 1B and C). Briefly, an alignment rod, which can be placed alongside the wall of the well periphery, was used as a distance and angle control. A stand support was also used to help maintain perpendicular alignment. Modified gene gun barrel was constructed by, and can be obtained from the MRC-LMB mechanical workshop. The distribution pattern was aimed to concentrate around the CA1 area of the hippocampus (Figure 1D). The affected diameter was previously determined to be approximately $3 \mathrm{~mm}[29]$.

\section{Survival assays and confocal microscopy}

OTBS were then cultured for a further $24 \mathrm{~h}$, or as otherwise noted. Lactate dehydrogenase (LDH) assay was used to determine cell survival following treatments according to manufacturers protocol (Clontech). Briefly, the colorimetric conversion of a tetrazolium salt added to the culture medium supernatant that absorbs at $490 \mathrm{~nm}$ is proportional to the number of lysed cells. The signal was normalized to untreated OTBS. Triton X-100 was used as a positive assay control to define maximal absorbance (i.e. equal to $0 \%$ survival). Survival was calculated as 1 - (sampled medium - negative control)/(positive control - negative control) and presented as a\%.

As the epicentre of biolistic delivery was aimed to the CA1 region, this regio-selective targeting was confirmed by EYFP signal. Targeting using the modified barrel was shown very reproducible from a $10 \mathrm{~mm}$ targeting distance using our method, yet any misaligned OTBS delivery was excluded from the subsequent analyses. Damaged cells were also identified using a Terminal deoxynucleotide transferase dUTP nick end labelling (TUNEL) assay (Click-iT ${ }^{\bullet}$ TUNEL Alexa Fluor Imaging Assay; Invitrogen) [46], which detects fragments of DNA from apoptotic and necrotic cells. dUTP solution was added to the OTBS as per manufacturers protocol. Propidium Iodide (PI) was also used to monitor the number of exposed nuclei. A final concentration of PI of $1.5 \mu \mathrm{M}$ was added to the OTBS and incubated for 30 min then washed three times with PBS.

The slices were subsequently fixed with $4 \%$ paraformaldehyde (PFA; Sigma-Aldrich). For lineage differentiation, antibodies rabbit anti-NeuN (Merck; MAB377) and guinea pig anti-GFAP (Abcam; 4674) at 1:1000 and 1:500 dilutions respectively were incubated with the OTBS for $1 \mathrm{~h}$. Following three PBS washes, the secondary donkey alexa fluor 488 anti-rabbit (Life technologies) and goat alexa fluor 547 anti-guinea pig (Life technologies) were used at 1:1000 and incubated $30 \mathrm{~min}$. Cells were counterstained with diamidino-2-phenylindole (DAPI) for 30 min then washed three times with PBS. Following corresponding labelling OTBS were mounted in Vectashield. Brain slices were examined using an upright Bio-Rad Radiance 2100 confocal microscope and the numbers of labelled cells were counted. Only comparative brain areas were evaluated between conditions and the same magnification, pinhole size (1 AU), and gain intensity were maintained for each test. Labelled cells were counted from confocal images taken completely within these brain regions.

\section{Statistical analysis}

Experiments were performed in at least three independent experiments. Results are presented as mean \pm standard deviation (SD). Data analysis was performed using Graphpad Prism 5.0 (La Jolla, USA). The unpaired 2-tailed Student's $t$ test was used for comparison of the average total amount of counted cells per representative image or the average absorbance measurements. A $p$ value of $<0.05$ was considered statistically significant.

\section{Additional file}

Additional file 1: Organotypic brain slice preparation using the vibroslicer. The brain mounted inside the DMEM-agarose matrix was glued onto the copper platform. The blade was then properly aligned as described in the methods section. Ice-cold PBS containing P/S was added to the recipient covering both the blade and imbedded brain. Once activated, the vibroslicer, at predetermined slicing amplitude and frequency, cuts OTBS of precise thickness. These OTBS, contrasted by the phenol red in the DMEM, can easily be seen. They are then lifted by the blade, scooped, and transferred to the appropriate medium.

\section{Competing interests}

John O'Brien holds patents on the improved gene gun barrel. US patent number 10/380, 452 and European patent number 01974517.3.

\section{Authors' contributions}

JOB carried out the biolistic transfections and brain slice experiments, helped design the study, and contributed to writing the manuscript. JA wrote the manuscript and helped design the study. Both authors read and approved the final manuscript.

\section{Acknowledgements}

This work was supported by the Medical Research Council Laboratory of Molecular Biology. Video was filmed and edited by the MRC LMB visual aids department. Vibroslicer and modified gene gun barrel was constructed by the MRC LMB mechanical workshop department. We would like to thank SC Lummis for her help with the manuscript.

Received: 18 September 2013 Accepted: 16 December 2013 Published: 19 December 2013

\section{References}

1. Millet LJ, Gillette MU: Over a century of neuron culture: from the hanging drop to microfluidic devices. Yale J Biol Med 2012, 85:501-521.

2. Lossi L, Alasia S, Salio C, Merighi A: Cell death and proliferation in acute slices and organotypic cultures of mammalian CNS. Prog Neurobiol 2009, 88:221-245.

3. Cho $S$, Liu D, Fairman D, Li P, Jenkins L, McGonigle P, Wood A: Spatiotemporal evidence of apoptosis-mediated ischemic injury in organotypic hippocampal slice cultures. Neurochem Int 2004, 45:117-127.

4. Pringle AK, Sundstrom LE, Wilde GJ, Williams LR, lannotti F: Brain-derived neurotrophic factor, but not neurotrophin-3, prevents ischaemia-induced neuronal cell death in organotypic rat hippocampal slice cultures. Neurosci Lett 1996, 211:203-206.

5. Ray AM, Owen DE, Evans ML, Davis JB, Benham CD: Caspase inhibitors are functionally neuroprotective against oxygen glucose deprivation induced CA1 death in rat organotypic hippocampal slices. Brain Res 2000, 867:62-69. 
6. Drexler B, Hentschke H, Antkowiak B, Grasshoff C: Organotypic cultures as tools for testing neuroactive drugs - link between in-vitro and in-vivo experiments. Curr Med Chem 2010, 17:4538-4550.

7. Pena F: Organotypic cultures as tool to test long-term effects of chemicals on the nervous system. Curr Med Chem 2010, 17:987-1001.

8. Birgbauer E, Rao TS, Webb M: Lysolecithin induces demyelination in vitro in a cerebellar slice culture system. J Neurosci Res 2004, 78:157-166.

9. Krassioukov AV, Ackery A, Schwartz G, Adamchik Y, Liu Y, Fehlings MG: An in vitro model of neurotrauma in organotypic spinal cord cultures from adult mice. Brain Res Brain Res Protoc 2002, 10:60-68.

10. Newell DW, Barth A, Papermaster V, Malouf AT: Glutamate and nonglutamate receptor mediated toxicity caused by oxygen and glucose deprivation in organotypic hippocampal cultures. J Neurosci 1995, 15:7702-7711.

11. Ostergaard K, Finsen B, Zimmer J: Organotypic slice cultures of the rat striatum: an immunocytochemical, histochemical and in situ hybridization study of somatostatin, neuropeptide $Y$, nicotinamide adenine dinucleotide phosphate-diaphorase, and enkephalin. Exp Brain Res 1995, 103:70-84

12. Rytter A, Cronberg T, Asztely F, Nemali S, Wieloch T: Mouse hippocampal organotypic tissue cultures exposed to in vitro "ischemia" show selective and delayed CA1 damage that is aggravated by glucose. J Cereb Blood Flow Metab 2003, 23:23-33.

13. Barateiro A, Domingues HS, Fernandes A, Relvas JB, Brites D: Rat Cerebellar Slice Cultures Exposed to Bilirubin Evidence Reactive Gliosis, Excitotoxicity and Impaired Myelinogenesis that Is Prevented by AMPA and TNF-alpha Inhibitors. Mol Neurobiol 2013, 8:8530-8537.

14. Maywood ES, Drynan L, Chesham JE, Edwards MD, Dardente H, Fustin JM, Hazlerigg DG, O'Neill JS, Codner GF, Smyllie NJ, et al: Analysis of core circadian feedback loop in suprachiasmatic nucleus of mCry1-luc transgenic reporter mouse. Proc Natl Acad Sci USA 2013, 110:9547-9552.

15. Baratta J, Marienhagen JW, Ha D, Yu J, Robertson RT: Cholinergic innervation of cerebral cortex in organotypic slice cultures: sustained basal forebrain and transient striatal cholinergic projections. Neuroscience 1996, 72:1117-1132.

16. Franke $\mathrm{H}$, Schelhorn $\mathrm{N}$, Illes $\mathrm{P}$ : Dopaminergic neurons develop axonal projections to their target areas in organotypic co-cultures of the ventral mesencephalon and the striatum/prefrontal cortex. Neurochem Int 2003, 42:431-439.

17. Mingorance A, Fontana X, Sole M, Burgaya F, Urena JM, Teng FY, Tang BL, Hunt D, Anderson PN, Bethea JR, et al: Regulation of Nogo and Nogo receptor during the development of the entorhino-hippocampal pathway and after adult hippocampal lesions. Mol Cell Neurosci 2004, 26:34-49.

18. Oishi Y, Baratta J, Robertson RT, Steward O: Assessment of factors regulating axon growth between the cortex and spinal cord in organotypic co-cultures: effects of age and neurotrophic factors. J Neurotrauma 2004, 21:339-356.

19. Woodhams $\mathrm{PL}$, Atkinson DJ: Regeneration of entorhino-dentate projections in organotypic slice cultures: mode of axonal regrowth and effects of growth factors. Exp Neurol 1996, 140:68-78.

20. Chuang HN, Lohaus R, Hanisch UK, Binder C, Dehghani F, Pukrop T: Coculture System with an Organotypic Brain Slice and 3D Spheroid of Carcinoma Cells. J Vis Exp 2013, 80. doi. 10.3701/50881.

21. Gahwiler BH: Organotypic monolayer cultures of nervous tissue. J Neurosci Methods 1981, 4:329-342

22. Lindner $\mathrm{G}$, Grosse $\mathrm{G}$ : Morphometric studies of the rat hippocampus after static and dynamic cultivation. Z Mikrosk Anat Forsch 1982, 96:485-496.

23. Stoppini L, Buchs PA, Muller D: A simple method for organotypic cultures of nervous tissue. J Neurosci Methods 1991, 37:173-182.

24. Kim H, Kim E, Park M, Lee E, Namkoong K: Organotypic hippocampal slice culture from the adult mouse brain: a versatile tool for translational neuropsychopharmacology. Prog Neuropsychopharmacol Biol Psychiatry 2013, 41:36-43.

25. Su T, Paradiso B, Long YS, Liao WP, Simonato M: Evaluation of cell damage in organotypic hippocampal slice culture from adult mouse: a potential model system to study neuroprotection. Brain Res 2011, 1385:68-76.

26. Legradi A, Varszegi S, Szigeti C, Gulya K: Adult rat hippocampal slices as in vitro models for neurodegeneration: studies on cell viability and apoptotic processes. Brain Res Bull 2011, 84:39-44.
27. O'Brien JA, Holt M, Whiteside G, Lummis SC, Hastings MH: Modifications to the hand-held Gene Gun: improvements for in vitro biolistic transfection of organotypic neuronal tissue. J Neurosci Methods 2001, 112:57-64.

28. Geiger JR, Bischofberger J, Vida I, Frobe U, Pfitzinger S, Weber HJ, Haverkampf $\mathrm{K}$, Jonas P: Patch-clamp recording in brain slices with improved slicer technology. Pflugers Arch 2002, 443:491-501.

29. O'Brien JA, Lummis SC: Nano-biolistics: a method of biolistic transfection of cells and tissues using a gene gun with novel nanometer-sized projectiles. BMC Biotechnol 2011, 11:66.

30. Staal JA, Alexander SR, Liu Y, Dickson TD, Vickers JC: Characterization of cortical neuronal and glial alterations during culture of organotypic whole brain slices from neonatal and mature mice. PLoS One 2011, 6:e22040.

31. De Simoni A, Griesinger CB, Edwards FA: Development of rat CA1 neurones in acute versus organotypic slices: role of experience in synaptic morphology and activity. J Physiol 2003, 550:135-147.

32. Pokorny J, Yamamoto T: Postnatal ontogenesis of hippocampal CA1 area in rats. II. Development of ultrastructure in stratum lacunosum and moleculare. Brain Res Bull 1981, 7:121-130.

33. Mewes A, Franke $H$, Singer D: Organotypic brain slice cultures of adult transgenic P301S mice-a model for tauopathy studies. PLoS One 2012, 7:e45017.

34. Daviaud N, Garbayo E, Lautram N, Franconi F, Lemaire L, Perez-Pinzon M, Montero-Menei CN: Modeling nigrostriatal degeneration in organotypic cultures, a new ex vivo model of Parkinson's disease. Neuroscience 2013, 256C:10-22.

35. Morin-Brureau M, De Bock F, Lerner-Natoli M: Organotypic brain slices: a model to study the neurovascular unit micro-environment in epilepsies. Fluids and barriers of the CNS 2013, 10:11.

36. Gerace E, Landucci E, Scartabelli T, Moroni F, Pellegrini-Giampietro DE: Rat hippocampal slice culture models for the evaluation of neuroprotective agents. Methods Mol Biol 2012, 846:343-354

37. Connelly CA, Chen LC, Colquhoun SD: Metabolic activity of cultured rat brainstem, hippocampal and spinal cord slices. J Neurosci Methods 2000, 99:1-7.

38. Laywell ED, Kearns SM, Zheng T, Chen KA, Deng J, Chen HX, Roper SN, Steindler DA: Neuron-to-astrocyte transition: phenotypic fluidity and the formation of hybrid asterons in differentiating neurospheres. J Comp Neurol 2005, 493:321-333.

39. Walder S, Zhang F, Ferretti P: Up-regulation of neural stem cell markers suggests the occurrence of dedifferentiation in regenerating spinal cord. Dev Genes Evol 2003, 213:625-630.

40. Lo CW: Transformation by iontophoretic microinjection of DNA: multiple integrations without tandem insertions. Mol Cell Biol 1983, 3:1803-1814.

41. Punkosdy GA, Blain M, Glass DD, Lozano MM, O'Mara L, Dudley JP, Ahmed $R$, Shevach EM: Regulatory T-cell expansion during chronic viral infection is dependent on endogenous retroviral superantigens. Proc Natl Acad Sci USA 2011, 108:3677-3682.

42. Judkewitz B, Rizzi M, Kitamura K, Hausser M: Targeted single-cell electroporation of mammalian neurons in vivo. Nat Protoc 2009, 4:862-869.

43. Livet J, Weissman TA, Kang H, Draft RW, Lu J, Bennis RA, Sanes JR, Lichtman JW: Transgenic strategies for combinatorial expression of fluorescent proteins in the nervous system. Nature 2007, 450:56-62.

44. Jammalamadaka A, Banerjee S, Manjunath BS, Kosik KS: Statistical analysis of dendritic spine distributions in rat hippocampal cultures. BMC Bioinformatics 2013, 14:287.

45. O'Brien J, Unwin N: Organization of spines on the dendrites of Purkinje cells. Proc Natl Acad Sci USA 2006, 103:1575-1580.

46. Gavrieli $Y$, Sherman $Y$, Ben-Sasson SA: Identification of programmed cell death in situ via specific labeling of nuclear DNA fragmentation. J Cell Biol 1992, 119:493-501.

\section{doi:10.1186/1756-0500-6-544}

Cite this article as: Arsenault and $O^{\prime}$ Brien: Optimized heterologous transfection of viable adult organotypic brain slices using an enhanced gene gun. BMC Research Notes 2013 6:544. 